\title{
Estado y políticas culturales en Argentina. Un análisis comparativo entre el Kirchnerismo y la Alianza Cambiemos (2007-2017)
}

\author{
State and cultural policies in Argentina. A comparative analysis between Kirchnerismo and Alianza Cambiemos \\ (2007-2017)
}

\section{Clarisa Inés Fernández}

Instituto de Investigación en Humanidades y Ciencias Sociales (IDIHCS). Facultad de

Humanidades y Ciencias de la Educación de la Universidad Nacional de La Plata. ,

Argentina

clarisainesfernandez@gmail.com

\begin{abstract}
RESUMEN:
El presente trabajo propone una aproximación analítica respecto del modo en que las gestiones gubernamentales nacionales de los últimos 10 años han promovido, planificado y desarrollado sus políticas culturales. Con ese objetivo, en un primer apartado se realiza un abordaje teórico respecto del campo de las políticas culturales. Luego, se avanza en una reconstrucción de las políticas culturales que se llevaron a cabo durante los dos gobiernos de Cristina Fernández de Kirchner (2007-2015) y los primeros dos años de la gestión de la Alianza Cambiemos (2015-2017). A partir de un análisis comparativo de las gestiones, se dará cuenta de los paradigmas culturales que subyacen a las mismas, y las modalidades de acción desarrolladas por el Estado en cada coyuntura particular. Concluiremos con la identificación de rupturas y continuidades entre dichas gestiones, recuperando la pregunta por el sentido de cultura que proponen y las tensiones que estos sentidos generan en el campo cultural nacional.
\end{abstract}

Palabras Clave: Políticas culturales, Argentina, Kirchnerismo, Cambiemos.

\section{Abstract:}

The present work is proposed as an analytical approach regarding the way in which the national governmental administrations of the last 10 years have promoted, planned and developed their cultural policies. With that objective, in a first section the ideas of cultural policy are theoretically worked on. Then, we advance in a reconstruction of the cultural policies that were carried out during the two governments of Cristina Fernández de Kirchner (2007-2015) and the first two years ofthe AlianzaCambiemos (2015-2017). Based on a comparative analysis, we will try to identify the cultural paradigms that underlie them and the modes of action developed by the State at each particular juncture will be analyzed. We will conclude with the identification of ruptures and continuities between these managements, recovering the question by the sense of culture that they propose and the tensions that these senses generate in the national cultural field.

KEYwORDs: Cultural policies, Argentina, Kirchnerismo, Cambiemos.

\section{INTRODUCCIÓN}

El presente trabajo ${ }^{1}$ es producto de nuestra investigación posdoctoral, la cual venimos desarrollando como resultado de trabajos anteriores, donde abordamos el análisis de la articulación entre dos campos: el de las políticas culturales y el del teatro comunitario argentino (Fernández, 2017,2018).Allí, dimos cuenta del vínculo complejo que existe entre las organizaciones artísticas comunitarias (específicamente los grupos de teatro comunitario) y el Estado, a partir de la disputa, la negociación y la apropiación de políticas públicas destinadas a la cultura impulsada desde el poder estatal. Nuestras reflexiones nos llevaron a indagar en las problemáticas que afloran desde las estructuras tradicionales del Estado cuando sus políticas se enfrentan con las dinámicas de los grupos comunitarios. Por otra parte, estas dificultades se ven afectadas por las concepciones que cada coyuntura política tiene de la idea de cultura.A partir de esas concepciones se 
desarrollan proyectos disímiles que, mayoritariamente, conciben a la cultura como un bien económicamente redituable, o se establecen juicios de valor basados en estereotipos de "alta cultura", que, lejos de estar caducos, continúan operando en la materialización concreta de esas políticas (Fernández, 2017).

A partir de estas reflexiones, nos interesa dar cuenta, en un análisis comparativo, de los modos en que el campo cultural se estructuró durante los últimos dos gobiernos kirchneristas (2007 a 2011 y 2011 a 2015) y los dos primeros años de la gestión de la Alianza Cambiemos (2015-2017). Analizaremos los sentidos que subyacen a las políticas culturales implementadas -respecto de la idea de cultura que se propugna-, con el fin de reflexionar sobre los procesos políticos concretos que se llevaron adelante en materia de cultura. Identificaremos limitaciones y potencialidades, actores en juego, disputas materiales y simbólicas, problemáticas históricas y coyunturales. Consideramos que, más allá del momento histórico seleccionado para el análisis, el presente trabajo puede ser considerado un aporte en el campo de los estudios comparativos, en tanto propone analizar el momento transicional -con sus continuidades y rupturas- entre un modelo de gestión basado en la fuerte presencia del Estado en la toma de decisiones y en la elaboración de políticas culturales, a un modelo de corte neoliberal más profundo, con mayor presencia del mercado y de los organismos internacionales.

El desarrollo del trabajo se propone de la siguiente manera. En un primer momento, abordaremos teóricamente la idea de políticas culturales, reconstruyendo distintas visiones y paradigmas. Luego, desarrollaremos un apartado en clave histórica, donde reconstruiremos los elementos fundamentales de los periodos históricos seleccionados, en una doble dimensión que buscará articular una mirada históricopolítica. Finalmente, propondremos un ejercicio de reflexión, donde realizaremos un análisis comparativo respecto de las políticas culturales que se llevaron adelante en los periodos propuestos, y las ideas de cultura que subyacen a dichas políticas.

\section{ENFOQUE TEÓRICO-METODOLÓGICO}

El presente artículo busca realizar una primera aproximación, a través del método del análisis histórico comparativo, al modo en que se diseñaron, planificaron e implementaron una serie de políticas culturales durante las últimas dos gestiones kirchneristas y los primeros años de la gestión de Cambiemos. Se trabajará a partir de un análisis interpretativo de datos que fueron registrados a partir de tcnicas de recolección cualitativas. Entre ellas se encuentran: entrevistas a funcionarios públicos de la gestiones kirchneristas, a funcionarios de la alianza Cambiemos y a gestores culturales comunitarios. Además, se reconstruirán las políticas culturales a partir de documentos oficiales, registros virtuales y notas de prensa. $^{2}$

Nuestro objetivo es realizar un aporte al campo de estudios de las políticas culturales, a partir de un análisis que aborde periodos y políticas concretas. Consideramos que, si bien se trata de un campo de estudios en expansión, es escaso el número de trabajos que se encargan de analizar en concreto gestiones gubernamentales desde la perspectiva específica de la política cultural.

En relación con el corpus de políticas elegidas, se verá que las mismas corresponden a diferentes dimensiones, actores y alcances. En relación al criterio de selección de dichas políticas, seleccionamos aquellas que implicaron un cambio en el organigrama oficial (como transformaciones de ministerios a secretarías). Luego, incorporamos la apertura y cierre de diversos programas culturales que dieron cuenta de una reorientación de las visiones de cultura y que afectaban, principalmente, a las organizaciones culturales comunitarias. También tendremos en cuenta el modo en que las distintas gestiones utilizaron los espacios públicos, en tanto esto nos habla de los públicos proyectados y las actividades consideradas legítimas para ser financiadas por el Estado.

El trabajo se presenta dividido en tres apartados El primero desarrollará distintas visiones y reflexiones respecto al campo de las políticas culturales, las concepciones teóricas que adoptamos y su relevancia para analizar el vínculo que establece el Estado con las organizaciones de la sociedad civil en materia de cultura. 
Elaboraremos una suerte de clasificación de las políticas culturales en función de su alcance territorial, su proyección política y su área de ejecución, cuyo objetivo es convertirse en un insumo para el análisis posterior. En el segundo apartado, realizaremos una reconstrucción en clave histórica de las políticas culturales llevadas adelante por las últimas dos gestiones gubernamentales del kirchnerismo, y por los primeros dos años de la gestión de la alianza Cambiemos. Finalmente, en el tercer apartado realizaremos un análisis históricocomparativo de dichas políticas, con el fin de analizar las distintas visiones de cultura que dichas políticas presentan.

\section{ESTADO Y POLÍTICAS CULTURALES: MÁS ALLÁ DEL ESPECTÁCULO}

En trabajos anteriores (Fernández, 2017) afirmamos que el Estado es un espacio que no está conformado solo por instituciones, sino también por ideas y una correlación de fuerzas políticas, "lo que hace que se constituya como campo de disputa política por la reproducción o transformación del orden social" (Barbosa Lima, 2014, p. 120). En ese sentido, en la sociedad capitalista el ordenamiento normativosimbólico constitutivo de la vida social es realizado por medio del Estado. Reconocer esto implica repensar la importancia que el mismo adquiere en la vida de las personas y en la construcción de su mundo de sentidos. También nos lleva a considerar otra idea de Lechner (1981), la que postula que a la vez que el Estado se constituye por medio de las relaciones sociales por otra parte las constituye;es objeto y sujeto a la vez. De ahí la importancia de tener en cuenta los sentidos que se construyen en las múltiples políticas públicas estatales, ya sea en su dimensión discursiva como en su puesta en práctica en el territorio.

Thwaites Rey y Ouvina señalan una serie de rasgos estructurales que atraviesan la constitución de los Estados en América Latina desde los tiempos de la colonia, y destacan que en estos territorios "emerge un poder político jurídicamente soberano sobre un conjunto de un determinado territorio, pero que no tiene relación orgánica con aquellas poblaciones sobre las que pretende gobernar” (2012, p. 68). Estos rasgos llevaron a una distintiva "precariedad institucional", con carácter subordinado y dependiente del mercado mundial. El poder del Estado es un producto contingente, de cambios en el equilibrio de las fuerzas políticas situadas dentro y fuera de él, que es necesario analizar en la particularidad de cada coyuntura. Dentro de la lógica estatal nos interesan particularmente los modos en que se gestan, diseñan, planifican e implementan las políticas culturales.

Los estudios sobre políticas culturales se han multiplicado en el transcurso de los últimos años, tanto en América Latina como en Europa (Orozco, 2007). Se han realizado investigaciones sobre la evolución de las políticas culturales (Mejía Arango, 2009; Nivón, 2011), sobre el vínculo entre políticas culturales y participación ciudadana (Escobar et al., 2001; Ferreño, 2014),sobre la incidencia de las políticas culturales en las desigualdades económicas y simbólicas (Grimson, 2014), sobre distintos aspectos que abordan procesos de institucionalización de la cultura (Tovar, 1994; Crespo, 2003), sobre casos de implementación de políticas culturales (Bonfil, 2002; Nivón, 2004, 2006), y sobre políticas gubernamentales concretas (López, 2001). Encontramos también reconstrucciones de la trayectoria conceptual del término, clasificaciones y discusiones en torno a los paradigmas que las sustentan (García Canclini, 1987; Brunner, 1987; Ochoa, 2002; Bayardo, 2008a, 2008b; Maccioni, 2002; Mato, 2002; Wortman, 2002, 2005 ;Logiódice, 2012), su utilización en el contexto latinoamericano y las diferencias con las cultural politics europeas (Yúdice, 2002).

Además, el campo de estudios de las políticas culturales en Argentina ha sumado un vasto corpus de trabajos, que han reflexionado tanto respecto de las categorías propias del campo como de dimensiones específicas y casos concretos. Tal es el caso de investigaciones como las de Mihal (2009), quien analiza el Plan Nacional de Lectura (PNL) en relación con las políticas culturales locales de cada territorio. Por su parte, las investigaciones de Sánchez Salinas (2017, 2018a, 2018b) han abordado el campo de las políticas culturales a partir de la pregunta por programas específicos de los organismos estatales de cultura, en relación con grupos de teatro comunitario de la provincia de Mendoza. Szpilbarg y Saferstein (2014) reflexionan sobre 
las transformaciones que el concepto de industria cultural ha sufrido desde que fuera acuñado por primera vez en los escritos de los pensadores de Frankfurt, hasta llegar a su institucionalización en las políticas culturales locales. Estos mismos reconstruyen el vínculo que el ámbito de la cultura ha tenido con la comunicación y la economía, resaltando especialmente el diálogo de la cultura con el marketing, o, en términos de Achugar, el proceso de "economización de la cultura y culturización de la economía" (Achugar en Szilbarg y Saferstein, 2014, p. 107).

Barbieri (2014) afirma que las políticas culturales durante el siglo XX han sido pensadas como "políticas de objetos”, invisibilizando a los sujetos y grupos sociales. En esa línea, para Lacarrieu y Cerdeirael campo de las políticas culturales se ha constituido en relación a ciertos consensos, como "la idea de la cultura como esencia o elevación" (2016, p. 19).

La centralidad que adquirió el tema de la cultura, tanto a nivel mundial como en Latinoamérica, proviene en gran parte de su instalación en las agendas de los organismos mundiales hacia fines de los años 80 , y más fuertemente a principios del 2000 (Infantino, 2008). Los años 80 significaron un giro muy importante de la política cultural: el giro hacia la economía, como nueva perspectiva de evaluación y de legitimación de la acción cultural pública.El modelo francés que analiza Urfalino (1996) es una referencia general para ver la lógica universal del proceso de disolución del paradigma clásico de la política cultural. Este propone la "democratización cultural" por un mayor "acceso", a partir de la difusión de "la 'alta cultura' en tanto cultura universalizada" (Lacarrieu y Cerdeira, 2016, p. 20). Coincidimos con las autoras en afirmar que dicho modelo -que en Argentina podemos identificar en algunas políticas culturales que destacan la gratuidad, masividad e impacto cuantitativo de los eventos culturales- se propone como estrategia de inclusión social pero tiene dos limitaciones: por un lado, el hecho de tener más acceso a ciertos productos culturales o eventos no garantiza la apropiación de los mismos, y, por el otro, esta misma condición puede incluso promover jerarquizaciones que fortalecen las desigualdades preexistentes (Lacarrieu y Cerdeira, 2016).

En trabajos anteriores vimos que "los 90 se caracterizaron por la dogmatización neoliberal de los mecanismos del mercado, el impacto de las nuevas tecnologías, la masificación del consumo cultural y la expansión de los grandes aparatos culturales" (Fernández, 2018, p. 449). Autores como Zimmer y Toeppler (1996) han estudiado los procesos por los cuales se desvirtúan los lineamientos de la política cultural tradicional, orientándose hacia objetivos de signo liberal. Rodríguez Morató destaca que:

Este proceso de deterioro y agotamiento de las políticas culturales tradicionales, de base nacional, ha tenido como contrapartida un paralelo realce de la política cultural local. Esta tendencia ha sido luego extraordinariamente potenciada por el surgimiento del paradigma de la ciudad emprendedora, en la medida en que este encontró en la cultura un terreno fértil en que desarrollarse (2005, p. 352)

Desde allí el estrecho vínculo entre cultura y economía transformó el modo en que los artistas -muchas veces llamados trabajadores de la cultura - se perciben y gestionan su actividad. Desde mediados de los 90 las áreas del arte y la cultura han pasado a estar al frente de la "nueva economía”. El trabajo de Mc Robbie resalta que existe una gran ilegibilidad del trabajo en el sector creativo y cultural, porque pasó a ser un campo abonado para la autodisciplina, donde la libertad está encorsetada. Para la autora

La máquina de la cultura y el despliegue utilitario de la creatividad como forma de transformar aquello que llamamos trabajo. La promoción del trabajo creativo ha terminado por ser una estrategia de despolitización, una manera de suprimir toda connotación política de la esfera laboral, que se ha reemplazado por la gratificación de las propias aspiraciones y expectativas y por la expresión de uno mismo (2009, p. 155).

De esa manera, el mercado también es un actor que participa e influye activamente en el diseño y planificación de las políticas culturales.

En Argentina, a principios del siglo XXI, comienza desarrollarse en el ámbito de las políticas culturales una "nueva retórica con eje en la creatividad" (Bayardo, 2016, p. 160). Con ciertas raíces en el mencionado proceso de economización de la cultura, esta retórica tiene su origen en el modelo anglosajón de fines de 
los 90. Sin embargo, también encontramos reminiscencias de algunos elementos que signaron a las políticas culturales a partir de los 70, las cuales, en líneas generales, proponen ubicar a la cultura como un paliativo o solución de problemáticas de índole social (Bayardo, 2016).

A partir de lo anterior, entendemos que las políticas culturales se configuran como terreno de luchas por el poder, en el quese dan mediaciones entre lo cultural y lo político (Logiódice, 2012), espacios donde se desarrollan conflictos, coaliciones, movilización de recursos, grados relativos de autonomía y poder (Oszlak y O’Donnell, 2007). Esta visión de las políticas culturales nos permite pensar que las mismas son espacios en donde se pueden habilitar o clausurar demandas por derechos colectivos. Grimson (2014) afirma que las políticas culturales comprenden lo cultural como constitutivo de la ciudadanía y los derechos culturales, tan relevantes como los económicos, sociales y políticos, porque funcionan como dispositivos de las relaciones de fuerza sociales vigentes en un contexto determinado (Ferreño, 2014).Las políticas culturales están sustentadas por determinados paradigmas culturales, que conciben la cultura de un modo particular. ${ }^{3}$ A través de estos paradigmas se pueden explorar las políticas culturales en relación con el Estado, quien interviene en la cultura porque de ese modo "está afectando los sistemas de producción, circulación, distribución y reconocimiento del sentido" (Margulis et al., 2014, p. 11). Ello no implica perder de vista las interacciones con otros agentes como las empresas y los grupos comunitarios, los cuales generalmente obran vinculados por acción o por omisión a las intervenciones del Estado, sus infraestructuras, sus medios de financiamiento, sus regulaciones y sus arbitrajes de los conflictos.

A continuación presentamos un cuadro con un ordenamiento y clasificación posible de las políticas culturales, que nos permitirá ver más claramente las diversas dimensiones que atraviesan las orientaciones de las políticas culturales.
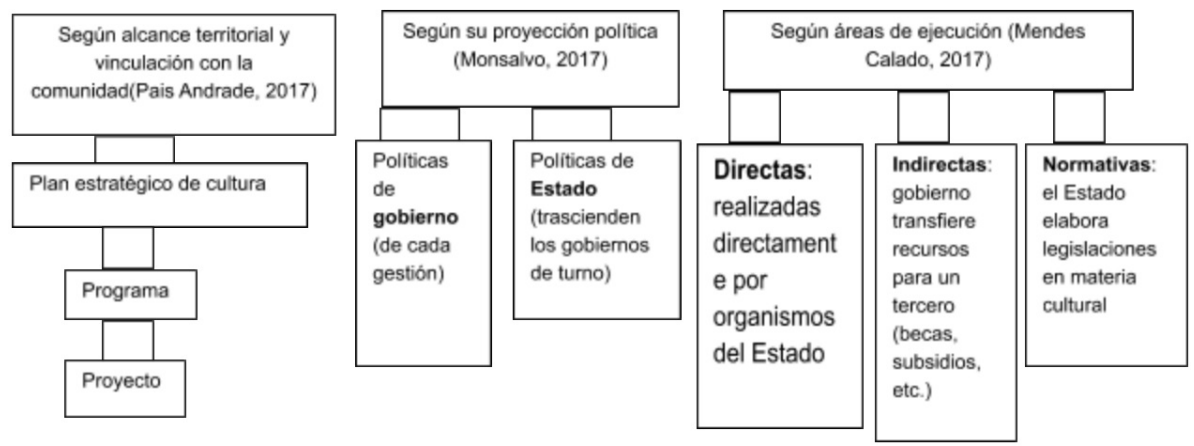

POLÍTICAS CULTURALES

Cuadro de elaboración propia que articula los aportes de los distintos autores citados

En el siguiente apartado, reconstruiremos en clave histórica una serie de políticas culturales que se desarrollaron entre los años 2007-2017, las cuales resultan significativas para pensar los sentidos de cultura subyacentes a cada gestión.

\section{BREVE RECONSTRUCCIÓN DE LAS GESTIONES CULTURALES (2007-2017)}

A continuación realizaremos una reconstrucción histórica que nos permitirá acercarnos al modo en que el Estado argentino ha venido desarrollando su política cultural. Nos interesa, particularmente, hacer una análisis comparativo entre las políticas culturales de los últimos dos gobiernos kirchneristas (2007 a 2011 y 2011 a 2015) y de los dos primeros años transcurridos desde la asunción de la alianza Cambiemos (2015-2017).

En Argentina, luego de atravesar la década del 90, el nuevo siglo trajo nuevos dilemas en materia cultural. En el escenario actual, la acción de las políticas culturales más visible fue la del Estado (Ochoa Gautier, 
2002), aunque otros actores, como el mercado, las organizaciones comunitarias, las empresas y las ONG, juegan un papel protagónico en la cadena de planificación, gestión e implementación de las políticas culturales (Fernández, 2017).

En un trabajo reciente, Segura y Prato (2018) aseguran que entre los años 2003 y 2015 existió en Argentina un "activismo estatal inédito" (p. 14) de un gran impacto social, que estuvo basado en el trabajo conjunto del Estado y las organizaciones y redes de la sociedad civil. En materia de cultura, las autoras destacan que los gobiernos kirchneristas dieron una "batalla cultural" (p. 44) que transformó aspectos de las diversas esferas de la cultura argentina. "Batalla cultural" fue una metáfora utilizada por el kirchnerismo para resignificar el ámbito de las políticas culturales con una nueva concepción del vínculo entre cultura y política.La misma metáfora es también compartida por sus funcionarios de otros países, como Gilberto Gil, quien fue ministro de Cultura desde 2003 a 2008 en Brasil (Lacarrieu y Cerdeira, 2016).

Desde nuestro recorte temporal, coincidimos con las autoras y con Zamorano (2016) en destacar ciertos aspectos de esas transformaciones, que significaron una democratización de la cultura y un fortalecimiento de los derechos culturales para sectores invisibilizados de la sociedad. Nos referimos a que dichas políticas ampliaron la definición de cultura desde la que se parte en relación con el Estado, y generaron una serie de estructuras administrativas que permitieron la expansión de la actividad cultural y un replanteo más profundo sobre la importancia de la misma como espacio de disputa de derechos. ${ }^{4}$ Pero, ¿de qué políticas estamos hablando?

Durante los años 2004-2009 fue secretario de Cultura el politólogo José Nun, quien llevó adelante una política basada en la idea de la democratización cultural. En el año 2006 se creó el Sistema de Información Cultural de la Argentina (SINCA), cuyo objetivo era recopilar información para la realización de estadísticas que distingan los diversos perfiles de consumo según las regiones del país. También se creó el Sistema de Información Cultural del Mercosur (SICSUR), que propuso la recolección y sistematización de datos culturales de los 10 países de América del Sur. Tanto el SINCA como el SICSUR se transformaron en bases de datos públicas que generaron estadísticas y publicaciones periódicas.

En el año 2009 se aprobó la Ley n 26.522 de Servicios de Comunicación Audiovisual (LSCA), y la creación de la Autoridad Federal de Servicios de organismo de Comunicación Audiovisual (AFSCA). Prato, Traversaro y Segura (2018) analizaron el proceso de elaboración de esta ley, y llegaron a la conclusión de que, en el marco de movilización y complejización social que había dejado la crisis del 2001, la necesidad de regular el debate suscitado por la ley -la cual tuvo una inusitada participación de la sociedad civil y de las organizaciones sociales- y la legitimidad lograda en el proceso coadyuvaron a la emergencia de un "incremento de la organización de la sociedad civil en torno a demandas regulatorias y financieras relativas a la música, danza, publicaciones independientes y producciones culturales comunitarias” (pp. 44-45). En ese sentido, la LSCA se erige como un antecedente relevante que "colocó a la comunicación y a la cultura en un lugar relevante de la agenda gubernamental” (p.45). En esa línea, las autoras analizan cuatro proyectos de leyes culturales que se presentaron en el Congreso de la Nación ${ }^{5}$ en los que se planteaban cambios en el vínculo entre las organizaciones y el Estado.Su aplicación generó un nuevo espacio de posibilidades de negociación y nuevas estrategias de parte de ambos actores.

Otra acción de la gestión kirchnerista fue la inauguración de Tecnópolis, en el año 2011, una megamuestra de arte, ciencia y tecnología (la más grande América Latina), que se realiza de julio a noviembre de cada año. El predio, ubicado en Villa Martelli, abría sus puertas gratuitamente a eventos culturales y muestras.

En el período 2006-2013 se realizaron cuatro congresos nacionales de cultura y dos congresos internacionales de cultura para la transformación social (en los cuales el gobierno nacional y los gobiernos provinciales tuvieron gran incidencia). Durante el año 2012 se debatió la Ley Federal de las Culturas (en la cual nos detendremos luego), la cual comenzó un recorrido administrativo que se vio interrumpido en el año 2015 con la asunción del nuevo gobierno. Otra iniciativa fue la creación del Programa Nacional de Puntos de Cultura (2011), sobre el cual nos detendremos particularmente, ya que su creación en Argentina está ligada 
al desarrollo de un programa de alcance regional, llamado Cultura Viva Comunitaria. Este tiene su origen en un proyecto brasilero surgido en el año 2004 , que propone que el $0,1 \%$ del presupuesto nacional se destine a gestionar actividades culturales de diversas organizaciones y agrupaciones sociales que trabajen vinculadas a la comunidad. Argentina presentó el proyecto análogo en el Congreso en el año 2012, y el colectivo Pueblo hace Cultura $^{6}$-una acción mancomunada de más de 90 grupos, radios, bibliotecas y diversas instituciones de todo el país-ha llevado adelante esta propuesta. El impulso de Puntos de Cultura en Argentina se realizó gracias a la gestión de los miembros de la Red de Teatro en Comunidad y otras organizaciones como El Culebrón Timbal o Crear Vale la Pena, que promovieron la visita a la Argentina de Celio Turino, creador de Puntos de Cultura en Brasil.A partir de ese evento comenzó a pensarse en la creación de la versión argentina de este programa (Fernández, 2018). Lo que el Programa ofrece puntualmente es financiamiento para proyectos culturales de base comunitaria, que tengan o no personería jurídica y que ya estén funcionando en su territorio.

Este programa,en tanto política cultural orientada a generar inclusión social y democracia cultural,ha sido objeto tanto de halagos como de críticas provenientes de la comunidad académica. Retomaremos estas discusiones en el último apartado.

Otros programas que se crearon en estos años fueron: el Programa de Soporte al Emprendimiento Cultural, el Plan Ceibo ${ }^{7}$ y el Plan Nacional de Igualdad Cultural. ${ }^{8}$ En el año 2014 se creó el Ministerio de Cultura, que estuvo a cargo de Teresa Parodi hasta diciembre del 2015. En mayo del 2015 se abrió el Centro Cultural Kirchner, un espacio cultural ubicado en el antiguo Palacio de Correo, que fue declarado como monumento histórico Nacional (Ley 12.662), y que se considera la obra de infraestructura cultural más grande América Latina. ${ }^{9}$

Durante la gestión kirchnerista tuvo lugar la presentación de dos iniciativas provenientes de distintos sectores de la sociedad civil, las cuales, a nuestro entender, dan cuenta de nuevas dinámicas de articulación con el Estado, vinculadas su concepción de cultura y a la participación ciudadana en procesos de planificación, gestión e implementación de las políticas culturales. Se trata de la presentación del proyecto de Ley Federal de las Culturas Comunitarias - que nombramos anteriormente- y del Consejo de Cultura de Hurlingham. Ambas propuestas se encuentran en instancias de negociación con el Estado para lograr cierto grado de institucionalización. Consideramos que ambas propuestas se inscriben en nuevas dinámicas de articulación con el Estado por los siguientes motivos: en primer lugar, se trata de iniciativas que surgen de actores de la sociedad civil que no están vinculados directamente con la gestión estatal. Este aspecto es fundamental porque rompe con la idea de un programa que sea instalado para que sea "aprovechado" por las comunidades. En ese sentido, el diseño, la planificación, los postulados que los sostienen y su modo de funcionamiento son ideados por la comunidad, más allá de que interpelan y prevén la participación estatal. Por otro lado, las visiones de cultura que propugnan estos proyectos incluyen la diversidad cultural, es decir, la participación de sectores históricamente invisibilizados como sujetos de derechos culturales, como los pueblos originarios, las minorías sexuales, entre otros.

La primera de las propuestas (Ley Federal de las Culturas), surgió del Frente de Artistas y Trabajadores de la Cultura (FAyTC), compuesto por personajes del mundo artístico, político e intelectual. La idea de este colectivo era elaborar una Ley Federal de las Culturas a través de la discusión de 21 puntos, para lo cual se convocó a distintas organizaciones y trabajadores de la cultura. Se planteaba que existieran dos consejos federales (uno de carácter resolutivo y otro consultivo), cuyas decisiones estarían articuladas con el Ministerio de Cultura, que sería el órgano de aplicación de la ley. El proyecto de ley fue presentado en el año 2015 a la entonces directora del ministerio, Teresa Parodi, pero con el cambio de gobierno no pudo cumplimentar su recorrido parlamentario. Por ese motivo, en junio del 2017 se volvió a hacer una presentación de la misma. Entre sus aspectos fundamentales,esta ley postula una idea de culturas en plural que tenga en cuenta la diversidad del territorio nacional. Por otra parte, el Estado aparece como una institución articuladora, que debe potenciar la actividad cultural a través del fortalecimiento de las producciones locales. A la presencia de un Consejo Federal de las Culturas, la ley agregaría a un representante de la CGT y a un organismo 
consultivo, de carácter no vinculante, que representara a los colectivos y organizaciones nacionales de artistas y trabajadores de la cultura.

La propuesta del Consejo de Cultura de Hurlinghames una iniciativa liderada por Jorge Pagés, un referente cultural de la localidad de Hurlingham, provincia de Buenos Aires, quien forma parte de una organización cultural llamada La Rotonda Cultural. ${ }^{10}$ La propuesta consiste en la elaboración de un plan estratégico de cultura para la localidad, que plantea la creación de un consejo de cultura. El fin último de este instrumento sería que los distintos sectores de la comunidad pudieran participar de la construcción de legislación en materia de cultura, como la elaboración de proyectos o líneas directrices para determinadas políticas. Además, es un área compartida entre el Estado y la sociedad civil, para poder cogestionar en determinadas áreas. El modelo del consejo fue tomado de la experiencia brasilera del Municipio de Río Branco, al noroeste de Brasil. Desde la gestión del gobierno de Lula Da Silva, con la dirección de Gilberto Gil y Jura Ferreira en el área de Cultura, se impulsó la creación de estos consejos comunitarios como una política de Estado. Existen aproximadamente mil cuatrocientos Consejos en Brasil, los cuales, si bien responden a una reglamentación general, cada uno adquiere características disímiles de acuerdo al territorio en el cual se establece. El Consejo de Río Branco, que es de tipo asambleario, permite la libre participación, en tanto que se abre un registro en el Municipio en el cual se anotan todos aquellos que están trabajando en el ámbito de la cultura y todos pueden ser consejeros. En Argentina, esta experiencia es novedosa, en tanto existen actualmente muy pocos casos que estén funcionando, de los cuales hay escasa información. El caso del Consejo de Hurlingham ha sido presentado en el Concejo Deliberante local, y si bien aún no hay definiciones oficiales, en el año 2018 se abrió una mesa de negociación con los representantes del poder ejecutivo local y concejales, para rever el proyecto y volver a presentarlo. ${ }^{11}$

A pesar del reconocimiento de lo que, a nuestro entender, fue el desarrollo de políticas culturales más inclusivas. Coincidimos con Zamorano (2016) en señalar que en la transformación de las políticas culturales en Argentina durante los años 2003-2013 aparecen también continuidades en relación a los elementos que históricamente caracterizaron a este ámbito: la falta de soporte presupuestario, la concentración de recursos en la Ciudad Autónoma de Buenos Aires y la inexistencia de una institucionalidad fuerte. Además, señala que las producciones fueron limitadas respecto al conjunto de las políticas sociales, lo que impidió dotar de jerarquía a las instituciones culturales.

En el año 2011 se creó el Mercado de Industrias Creativas Argentinas (MICA), cuyo funcionamiento responde a la lógica de "ciudad creativa" que describimos más arriba, y que se verá continuado y profundizado con la política cultural de Cambiemos. A la vez, los principales organismos nacionales de financiación de la cultura, como el Fondo Nacional de las Artes o el Instituto Nacional de las Artes, proponían (y aún lo hacen) líneas de subsidios que operan bajo la lógica del mérito y el talento como valores destacados.

Con la asunción de la alianza Cambiemos, en diciembre del 2015, se modificó la orientación de las políticas culturales que venía acompañando el periodo político anterior. Desde la asunción del nuevo ministro de Cultura, Pablo Avelluto, se dieron una serie de transformaciones, tanto hacia adentro del organigrama estatal, como hacia afuera, respecto de las políticas culturales implementadas. Entre estas transformaciones podemos puntualizar las modificaciones sustanciales que se realizaron en la Ley de Servicios de Comunicación Audiovisual, ${ }^{12}$ la apertura de importaciones -que afectó notoriamente a la industria en general y a la industria editorial en particular- ${ }^{13}$, y los centros culturales, muchos de los cuales sufrieron clausuras o desalojos. ${ }^{14}$ Además, se aprobó el Régimen de Promoción Cultural (Ley de Mecenazgo) ${ }^{15}$ provincial, que ya venía funcionando en la Ciudad de Buenos Aires desde el año 2006, la cual profundiza la participación del sector privado en el financiamiento de la cultura a partir de un criterio de selección que beneficia a las agrupaciones artísticas con mayor trayectoria.

Según los datos que hemos reconstruido (que deberán ser profundizados en tanto no existe una base de información oficial al respecto), hay diez programas que continúan vigentes de la administración 
kirchnerista a nivel nacional, y se crearon 15 programas nuevos durante la gestión de Cambiemos. Algunos de esos programas son: Academia Argentina, emprende + cultura (que brinda herramientas teóricas para el desarrollo de emprendimientos culturales), Gestión cultural pública (destinado a la profesionalización de agentes culturales del ámbito público federal), Red de ciudades creativas (destinado a municipios), Entornos creativos (destinado a escuelas públicas), entre otros.

En relación al presupuesto destinado a cultura, este sufrió un recorte del $12.6 \%$. Según un informe elaborado a partir de datos oficiales, ${ }^{16}$ durante el año 2016 el Ministerio de Cultura subejecutó su presupuesto en un $28 \%$, lo que equivale a decir que del total de recursos destinados para ese ministerio, solo se utilizó el $72 \%$ de dichos recursos. Esta subejecución podría implicar que al año siguiente los programas que sufrieron el recorte presupuestario ya no existieran, o se vieran como deficientes, lo que justificaría su desaparición.

Según los datos elaborados por el periodista Damián Fresolone, publicados en su página Cultura en Números, ${ }^{17}$ la subejecución del Ministerio de Cultura alcanzó un monto de $\$ 837.000 .000$, cifra que conjuga el dinero no utilizado por los programas ${ }^{18}$ del Ministerio y por otros entes dependientes de Pablo Avelluto, como el Teatro Nacional Cervantes, la Biblioteca Nacional, el Instituto de Teatro y el Fondo Nacional de las Artes.

En relación al vínculo del Estado con el sector artístico, podemos observar un aumento de la conflictividad, ya sea por el cierre de centros culturales - un caso paradigmático es el del Centro Cultural Olga Vázquez (ciudad de La Plata, Provincia de Buenos Aires), que implicó el veto de una ley ya sancionada ${ }^{19}$ - como por el recorte presupuestario que alcanzó a distintas áreas (nacionales, provinciales, municipales). Dentro de los casos más recientes, se encuentra el del Consejo Provincial de Teatro Independiente (dependiente del entonces Ministerio de Gestión Cultural de la Provincia de Buenos Aires), que se movilizó en un reclamo por la falta de pago de los subsidios y la desregulación de su mecanismo de funcionamiento. También, en diciembre del 2017 se descontinuó la financiación de la Compañía de Danza por la Inclusión, el ballet nacional dirigido por Iñaki Urlezaga, un reconocido artista argentino. El ballet contaba con 80 personas entre artistas, técnicos y vestuaristas.

Por otro lado, dos espacios culturales emblemáticos de la etapa kircherista se vieron intervenidos con la llegada de la nueva gestión. El titular del Sistema de Medios Hernán Lombardi, a cargo del Centro Cultural Kirchner (CCK) y de Tecnópolis, anunció a principios del 2016 que el 85 por ciento de los empleados delCCK serían despedidos. A su vez, dentro del parque Tecnópolis se desmanteló el área temática destinada a Zamba, un personaje creado por el canal infantil PakaPaka, que reconstruía la historia latinoamericana. Además, se despidieron unas 100 personas que trabajaban en este predio y comenzó a alquilarse el lugar para eventos privados.

Una acción destacada de Cambiemos, que va a contramano de las enumeradas anteriormente, la observamos en la creación en el año 2016 del Consejo Cultural Comunitario y de la Comisión Nacional de Puntos de Cultura (Segura y Prato, 2018). Destacamos estas acciones porque consideramos que el Programa Puntos de Cultura, que ha sido analizado por diversos investigadores y funcionarios pertenecientes al mismo programa (Wortman, 2017; Benhabib, 2018; Sánchez Salinas, 2018, entre otros), fue pensado desde una lógica diferente a los programas tradicionales. Si bien el programa surgió a partir de un modelo homónimo de Brasil, y estaba atado a un la decisión política estatal en cuanto a la disponibilidad de presupuesto y modalidades de participación, ciertas ideas que lo sustentan promueven el desarrollo de proyectos que ya están funcionando en el territorio, y que son planificados y sostenidos por las comunidades.

Lacarrieu y Cerdeira (2016) se preguntan si los programas como el de Puntos de Cultura realmente potencian el poder de estos colectivos o los retifican en sus lugares de subalternidad. Para incorporar a este debate referencian a Rubim, quien pone en duda la posibilidad de que los mismos puedan contribuir realmente a transformar el Estado, ya que sostienen un déficit de ciudadanía cultural. En ese sentido, afirman que “resulta imposible pensar la democracia cultural sin una 'radicalización' de la democracia y de los estados en sus diferentes niveles" (Rubim, p. 27).Por ello, si bien es necesario mantener una mirada atenta respecto 
del grado de autonomía que los colectivos culturales manejan con esta iniciativa, también es importante destacar que el Programa Puntos de Cultura está destinado a organizaciones que fueron muchas veces marginalizadas por no cumplir requisitos básicos (como la personería jurídica). Además, potencia con su financiación proyectos que ya están funcionando en la comunidad sin direccionar los ejes que lo estructuran, y plantea una dinámica en donde existe un diálogo permanente con las organizaciones interpeladas.

Hasta aquí, reconstruimos una serie de políticas estatales que tuvieron lugar en los periodos seleccionados. En el siguiente apartado retomaremos en clave analítica dichas políticas con el fin de reflexionar en torno a los modelos de cultura que cada gestión desarrolló.

\section{Modelos CUlturales: RUPTURA Y TRADiCión}

Las políticas públicas, en su concepción tradicional, suponen su desarrollo a partir de problemáticas que surgen del cuerpo social, pero si evaluamos con ojo crítico los programas elaborados por el Estado en materia de cultura, vemos que este supuesto diálogo entre políticas culturales y necesidades sociales es muy reducido.

En primer lugar, notamos una diferenciación numérica en cuanto a la cantidad de iniciativas desplegadas en la gestión kirchnerista y en la de Cambiemos. El gobierno nacional de la gestión Cambiemos ha signado su discurso público en la idea de "ajuste" y "ahorro", los cuales afectaron distintas áreas de la vida social. La cultura y la educación fueron particularmente intervenidas en sus presupuestos, lo que implicó cierre de programas, la reconfiguración de la estructura administrativa, y, específicamente, una transformación en la orientación de las políticas culturales. Delimitamos, así, dos paradigmas culturales que operan en las gestiones gubernamentales observadas. Es claro que el gobierno de Cambiemos quiso diferenciarse y marcar una ruptura con el kirchnerismo. En el área de Cultura esto se observa en varios aspectos: el borramiento de la historicidad de los programas y planes culturales que venían de la gestión anterior, la creación de nuevos programas, la modificación de la jerarquía de las dependencias estatales(transformación de secretarías en programas, desaparición de áreas, etc.). Estas transformaciones permiten ver el movimiento fluido del poder y las burocracias, y la institucionalidad débil (Jessop, 2014) que portan ciertas áreas del Estado.

Si volvemos al cuadro que elaboramos en el apartado anterior, vemos que en ambas gestiones hay preeminencia de programas como formato administrativo seleccionado. Los programas, al no ser parte de la estructura del Estado, son maleables y corren riesgo constante de vaciamiento y de desfinanciación por parte de las gestiones. A su vez, los mismos están sujetos a políticas de gobierno y no se transforman en políticas de Estado. Según sus áreas de ejecución, la mayor parte de las políticas culturales nacionales fueron acciones directas del Estado, aunque encontramos algunos casos como la Ley de Mecenazgo, que terceriza el acceso a los recursos.

Durante la gestión kirchenista se observa una mayor injerencia del poder ejecutivo en el proyecto cultural, a partir de la creación de la Unidad Bicentenario (2008), que respondía directamente a Cristina Fernández de Kirchner. Esta estaba a cargo de Javier Grosman, ${ }^{20}$ quien, además de los festejos por el 200 aniversario de la nación, sumó la creación de Tecnópolis y del Centro Cultural Kirchner, ambos edificios emblemáticos de la gestión. Estas acciones dan cuenta de un Estado que jerarquiza y potencia la producción de ciertos rasgos identitarios nacionales y latinoamericanistas en su política cultural. La dimensión simbólica se estructura fuertemente en la construcción de un mensaje de nación y de patria grande ${ }^{21}$ que caracterizó a los gobiernos kirchneristas. En relación al Centro Cultural Kirchner, Lacarrieu y Cerdeira (2016) afirman que es un edificio construido en el marco de un relato con un fuerte sentido de apropiación por parte de los ciudadanos, que, sin embargo, no tiene en cuenta a los sujetos, en tanto está pensado desde lo que Barbieri denomina como "política de los objetos" (Barbieri, 2014). Como afirmamos más arriba, el hecho de construir edificios, monumentos y espacios culturales no implica de por sí que los ciudadanos se reapropien del sentido o la significación -o más aún, el uso- que se proyectó para ese espacio. 
Así, si bien parecería que el ideario kirchnerista se opone al de Cambiemos porque apuesta por iniciativas de índole colectiva, en realidad vemos que a lo largo de las gestiones ese ideario convivió con el de la creatividad, el esfuerzo y la meritocracia individuales. Llegamos a esta conclusión al analizar propuestas como el MICA, o la lógica de funcionamiento de los subsidios otorgados por los entes de financiamiento de la cultura, en donde aparece en su plenitud la dimensión económica de la cultura, y el modo en que la promoción de la creatividad en ocasiones despolitiza la práctica artística y precariza la situación de los trabajadores de la cultura. Por otro lado, no podemos desconocer que, incluso desde este perfil mercantil, la emergencia del MICA contribuyó a una mayor democratización en la circulación de los bienes culturales en distintas regiones.

En relación a la dimensión normativa de las políticas culturales, observamos que las propuestas más innovadoras las encontramos durante la gestión kirchnerista con iniciativas provenientes de distintos sectores de la sociedad civil, como los que señalamos en el apartado anterior: Ley Federal de las Culturas y Consejos de Cultura. Creemos que ambas propuestas:

Se pueden considerar nuevos instrumentos para disputar no solo recursos materiales y voz en la elaboración de políticas culturales, sino también esquemas de pensamiento, visiones y concepciones de la cultura, que redundarán, posteriormente, en distintos procesos de participación ciudadana (Fernández, 2017, p. 14).

En esa línea, estas propuestas parten de dos postulados que quiebran con la dinámica tradicional del Estado que veníamos viendo (de creación de programas), porque se proyectan más allá de las gestiones y pretenden convertirse en políticas de Estado. Además, superan los límites de los proyectos territorialmente acotados para incorporar un alcance extendido y amplio de intervención, incorporando la diversidad cultural propia de nuestro país. Finalmente, en cuanto a las áreas de ejecución, el Estado es pensado como un órgano que institucionaliza estas propuestas, pero que legisla conjuntamente con distintas organizaciones de la sociedad civil. En función de esto, podemos afirmar que estas propuestas incluyen al ciudadano como partícipe de la elaboración de legislación en materia de cultura.

Finalmente, es fundamental revisar nuevamente el concepto de cultura que subyace a las políticas culturales de cada gestión, en tanto allí reside el punto neurálgico que tomará cuerpo en la operacionalización de distintas acciones gubernamentales. En función de lo analizado, podemos afirmar que en los periodos trabajados, desde todas las gestiones gubernamentales se evidencia un quiebre representativo respecto de lo que se propuso desde las políticas culturales estatales y las poblaciones a donde esas políticas iban destinadas. Las formas administrativas que adquirieron esas políticas fueron mayoritariamente programas, de corta duración, que se fueron modificando con la llegada de las nuevas gestiones al poder, y no estuvieron configuradas desde los espacios cotidianos donde se experimenta y construye la vida cultural, sino desde ámbitos fragmentados del Estado, desconectados entre sí, que propusieron campos de intervención específicos (danza, teatro, editoriales, entre otros) sin tener en cuenta las demandas localizadas de esas poblaciones.

A pesar de estas coincidencias, a lo largo del texto identificamos una diferencia notable en el vínculo que el Estado construyó con los distintos sectores de la sociedad civil durante las gestiones analizadas. Durante el kirchnerismo, la construcción discursiva y la modalidad de intervención de las políticas culturales estuvo en sintonía con el proyecto ideado por el Frente para la Victoria, orientado a recuperar y jerarquizar ciertos sectores invisibilizados de la cultura nacional, y a construir un imaginario basado en la idea de patria grande, y de unión latinoamericana. Si bien durante dichas gestiones hubo una proliferación de centros culturales, propuestas artísticas y, particularmente desde el Estado, una búsqueda por la sistematización de experiencias que redundara en la creación de una base de datos inédita hasta el momento, podemos ver una continuidad respecto de ciertas cuestiones que tradicionalmente signaron la estatalidad argentina: un bajo presupuesto en cultura, una estructura jerárquica basada en la figura fuerte del liderazgo (en este caso, de Cristina Fernández de Kirchner), y la planificación de megaproyectos que no responden a demandas de la población, sino a la construcción de emblemas materiales y simbólicos de la gestión. Además, como ya dijimos, este paradigma 
convivió con un acceso limitado a los recursos del Estado, en tanto se financiaron en mayor número proyectos que se sustentaban en los eslóganes de la "creatividad" y el emprendedurismo vinculados fuertemente al mercado de la industria cultural económicamente redituable. Identificamos, entonces, que tal como afirman Lacarrieu y Cerdeira (2016, p. 25), "en el kirchnerismo convivieron diferentes modelos: la 'política de los objetos', la política del acceso y la democratización cultural, y las políticas de la democracia cultural (como Puntos de Cultura)".

Estas continuidades se vieron profundizadas y complejizadas con la llegada de la alianza Cambiemos,momento a partir del cual el sector cultural y artístico se vio concretamente perjudicado en el desarrollo de sus actividades y proyectos. Se profundizó así la reducción del presupuesto, se clausuraron espacios y comenzó un periodo de fuerte borramiento de todo aquello que había surgido en la gestión anterior, más allá de lo que esas propuestas habían podido generar en los territorios. Los mecanismos estatales se transformaron y las jerarquías hacia adentro de las dependencias comenzaron a regirse por nuevas reglas. ${ }^{22}$ Los criterios que guían las políticas culturales desde diciembre del 2015 reivindican la idea de "alta cultura",la cual, con ciertos matices, avances y retrocesos, se ha mantenido a lo largo de los años.

Cambiemos despliega un modelo de políticas culturales entre emprendedor y neoliberal, que tiene bases teóricas en el mundo anglosajón y que se basa en el concepto de creatividad. Tal como afirma Bayardo, "las industrias creativas aparentan involucrar políticas culturales, pero más bien desdibujan los contornos emanados de su cometido en los derechos humanos y en la realización progresiva de la ciudadanía" (2016, p. 171).

La postura de Bayardo coincide con la de Lacarrieu y Cerdeira (2016), quienes identifican en la política cultural de Cambiemos una recolocación del Estado en el papel de "facilitador" (p. 30), dado que no aparecen propuestas desde lo estatal que se comprometan con la construcción de una democracia cultural inclusiva, y tampoco la apuesta por crear una sentido más participativo de la cultura.

Consideramos, finalmente, que las propuestas culturales que surgen desde otros sectores de la sociedad civil (como la de la Ley Federal de las Culturas y los Consejos Comunitarios) aportan una nueva forma de concebir a la cultura, en la que se revierte la jerarquía, se piensan propuestas que surgen de las vivencias cotidianas de la diversidad que conforma el cuerpo social nacional, donde el poder no es concebido como burocracia, y donde, principalmente, no se desconoce el poder del Estado como institución central de la vida social, sino que se busca una instancia de cogestión entre él y los distintos sectores sociales. En esa línea, Yúdice destaca el paradigma de la Cultura Comunitaria como una base para la creación de políticas realmente democráticas, en tanto "no se trata de operar con herramientas ya hechas del Estado, sino de diseñarlas desde una ciudadanía plural y participativa” (2018, p. 11).

\section{A MODO DE CIERRE}

A lo largo de nuestro análisis identificamos una serie de agentes que intervinieron fuertemente en las gestiones de cada gobierno, lo que da cuenta de la dimensión contingente y cambiante del Estado, y de la existencia de un equilibrio de fuerzas situadas dentro y fuera de él (Jessop, 2014). Así, reconocimos figuras políticas fuertes, que van desde el ejecutivo al legislativo, organizaciones de la sociedad civil y organismos internacionales, que configuran esas prácticas micropolíticas que estructuran cotidianamente la vida estatal, fundamentalmente respecto del ámbito cultural.

Este trabajo pretendió ser un aporte al campo de las políticas culturales, como una primera aproximación al análisis concreto del modo en que las mismas fueron planificadas e implementadas en los últimos diez años. A su vez, más allá del periodo espacio-temporal seleccionado, el presente trabajo buscó constituirse en un aporte significativo al campo de los estudios comparativos referidos a los modelos de gestión estatales, sus criterios decisionales y dinámicas de funcionamiento. Esa pretensión estuvo enmarcada en un intento por reconocer las dinámicas estatales propias de nuestra región. La comparación entre las gestiones buscó dar cuenta de 
cómo operan distintas visiones de cultura en las políticas que se desarrollaron, y los modos en que los mismos habilitaron o censuraron procesos de los distintos sectores que conforman la vida cultural nacional.

\section{ReFERENCIAS}

Barbieri, N.,Partal, A., y Merino, E. (2011). Nuevas políticas, nuevas miradas y metodologías de evaluación. ¿Cómo evaluar el retorno social de las políticas culturales? Papers, Revista de Sociología, 96(2), 477-500.

Barbieri, N. (2014). Cultura, políticas públicas y bienes comunes: hacia unas políticas de lo cultural. Revista Ágora, $1(1), 101-119$.

Barbosa Lima, L. (2014). As políticas culturais como espaço de intervenção crítica dos estudos culturais. En A. Grimson (Ed), Culturas politicas y politicas culturales (pp. 117-130). CABA, Argentina: Ediciones Böll Cono Sur.

Bayardo, R. (2008a). Políticas culturales: derroteros y perspectivas contemporáneas, RIPS. Revista de Investigaciones Politicas y Sociológicas, 7(1), 17-29.

Bayardo, R. (2008b). ¿Hacia dónde van las políticas públicas culturales? $1^{\circ}$ Simposio Internacional de Políticas Públicas Culturales en Iberoamérica, Facultad de Ciencias Económicas de la Universidad Nacional de Córdoba, Córdoba.

Bayardo, R. (2016). Creatividad y políticas culturales públicas en la Ciudad de Buenos Aires a comienzos del siglo XXI. Etnografias Contemporáneas, 2(3), 160-174.

Benhabib, D. (2018). Puntos de Cultura. Dinámica de lo impensado. En V.Prato y S.Segura (Ed), Estado, sociedad civil y politicas culturales. Rupturas y continuidades en Argentina entre 2003 y 2017 (pp. 231-256). Buenos Aires: RGC Ediciones.

Bonfil Batalla, G. (2002). Culturas populares y politica cultural. México: CONACULTA.

Brunner, J. (1987): Ciencias sociales y el tema de la cultura: notas para una agenda de investigación. Documento de Trabajo $\mathrm{N}^{\circ}$ 332. Santiago de Chile: FLACSO.

Crespo Oviedo, L. F. (2003). Políticas culturales: viejas tareas, nuevos paradigmas. Derecho y Cultura, 9, 23-42.

Escobar, A., Álvarez, S., y Dagnino, E. (2001). Política cultural y cultura política. Una nueva mirada sobre los movimientos sociales latinoamericanos. Madrid: Taurus e ICANH.

Fernández, C. (2017). Políticas culturales y organizaciones comunitarias. Coordenadas metodológicas para un análisis desde la complejidad. Ponencia presentada en las XII Jornadas de Sociología de la UBA. Universidad Nacional de Buenos Aires, Ciudad de Buenos Aires.

Fernández, C. (2018). Políticas culturales en acto. Teatro comunitario argentino: entre el Estado y la autogestión. Papers, Revista de Sociología, 103(3), 447-477.

Fernández, L. (2014). En nombre de los otros. Ciudadanía y políticas culturales. En A.Grimson (Comp). Culturas politicas y politicas culturales (pp. 109-116). CABA: Ediciones Böll Cono Sur.

García Canclini, N. (Ed.) (1987). Políticas culturales en América Latina. México: Grijalbo.

Grimson, A. (2014). Políticas para la justicia social. En A. Grimson (Comp.),Culturas políticas y políticas culturales (pp.9-14). CABA: Ediciones Böll Cono Sur.

Infantino, J. (2008). El arte como herramienta de intervención social entre jóvenes en la ciudad de Buenos Aires. La experiencia de Circo Social del Sur. Medio Ambiente y Urbanización, 69(1), 35-54.

Jessop, B. (2014). El Estado y el poder. Utopia y Praxis Latinoamericana, Revista Internacional de Filosofia Iberoamericana y Teoria Social, 66, 19-35.

Lacarrieu, M., yCerdeira, M. (2016). Institucionalidad y políticas culturales en Argentina. Límites y tensiones de los paradigmas de democratización y democracia cultural. Politicas Culturales Revista, 9, 10-33.

Lechner, N. (1981). Acerca del ordenamiento de la vida social por medio del Estado. Revista Mexicana de Sociología, 3(3), 1079-1102.

Logiódice, M. J. (2012). Políticas culturales, la conformación de un campo disciplinar. Sentidos y prácticas en las opciones de políticas. Documentos y aportes en administración pública y gestión estatal, 18, 59-87. 
López Borbón, L. (2001). Politicas culturales orientadas al plano de la vida cotidiana: evaluación de las estrategias de comunicación del Programa de Cultura Ciudadana (Bogotá 1995-1997). Argentina: CLACSO.

Maccioni, L. (2002). Valoración de la democracia y resignificación de 'política' y 'cultura': Sobre las políticas culturales como metapolíticas. En D.Mato (coord.), Estudios y otras prácticas intelectuales latinoamericanas en cultura y poder (pp. 189-200).Caracas: CLACSO.

Margulis, M., Urresti, M., y Lewin, H. (Comp.) (2014). Intervenir en la cultura: más allá de las políticas culturales. Buenos Aires: Biblos.

Mato, D. (2002). Estudios y otras prácticas intelectuales latinoamericanas en cultura y poder. En D.Mato (coord.), Estudios y otras prácticas intelectuales latinoamericanas en cultura y poder [21-48]. Caracas, Venezuela: CLACSO.

McRobbie, A. (2009). Industria cultural. MACBA, Ideas recibidas. Un vocabulario para la cultura artística contemporánea. Barcelona: MuseuD'ArtContemporani de Barcelona.

Mejía Arango, J. (2009). Apuntes sobre las políticas culturales en América Latina, 1987- 2009. Colombia Pensamiento Iberoamericano: Revista de Economía Politica,4, 5-130.

Mendes Calado, P. (2017). Pensamiento estratégico y metodologías para asumir los nuevos desafíos culturales. Gestión Cultural Pública. Coordenadas, herramientas y proyectos. Publicación digital la Dirección Nacional de Formación Cultural del Ministerio de Cultura de la Nación.

Mihal, I. (2009). Plan nacional de lectura: notas sobre una política de promoción de la lectura. Revista Pilquen, 11(1), $1-9$.

Monsalvo, M. (2017). (Re) pensar las políticas culturales desde una perspectiva pública. Gestión Cultural Pública, coordenada, herramientas y proyectos. Publicación digital la Dirección Nacional de Formación Cultural del Ministerio de Cultura de la Nación.

Nivón Bolán, E. (2004). Malestar en la cultura. Conflictos en la política cultural mexicana reciente. Pensar Iberoamérica, revista de cultura, 7, recuperado de https://www.oei.es/historico/pensariberoamerica/ ric07a01.htm

Nivón Bolán, E. (2006) Politicas culturales en México: 2006-2020. Hacia un plan estratégico de desarrollo cultural. México: Universidad de Guadalajada- Miguel Ángel Porrúa.

Nivón Bolán, E. (2011).As políticas culturais e os novos desafios. O patrimônio imaterial na estruturação das novas políticas culturais. En L. Calabre(Coord.), Politicas Culturais, teoría e práxis [40-61]. Rio de Janeiro: Fundação Casa de Rui Barbosa.

Ochoa, A.M. (2002). Políticas culturales, academia y sociedad. En D. Mato, (coord.) Estudios y otras prácticas intelectuales latinoamericanas en cultura y poder (pp. 213-224). Caracas: Consejo Latinoamericano de Ciencias Sociales y CEAP.

Orozco, J. L. (2007). Políticas culturales y modelos de gestión cultural. En Políticas culturales. Una revisión desde la gestión cultural [19-44]. México: Sistema de Universidad Virtual.

Ozlak, O. y O 'Donell, G. (2007). Estado y políticas estatales en América Latina: hacia una estrategia de investigación. En Lecturas sobre el Estado y las políticas públicas. Retomando el debate de ayer para fortalecer el actual [99-128]. Proyecto de modernización del Estado. Jefatura de Gabinete de Ministros de la Nación. Buenos Aires.

Pais Andrade, M. (2014). Transformar (se) en y desde la intervención cultural, Gestión Cultural Pública, coordenadas, herramientas y proyectos. Publicación digital la Dirección Nacional de Formación Cultural del Ministerio de Cultura de la Nación.

Prato, V., Traversaro, N. G., y Segura, S. (2018). La participación de la sociedad civil en las nuevas normas culturales. De la Ley de Servicios de Comunicación Audiovisual al Proyecto de Ley Federal de las Culturas. En V.Pratoy S. Segura(Ed),Estado, sociedad civil y politicas culturales. Rupturas y continuidades en Argentina entre 2003 y 2017 (pp. 43-65). Buenos Aires: RGC Ediciones.

Rodriguez Morató, A. (2005). La reinvención de la política cultural a escala local: el caso de Barcelona. Sociedade e Estado, 2(20), 337-350. 
Sánchez Salinas, R. (2017).El teatro comunitario en el proceso de transformación de la sociedad: el caso de Res o no Res en el barrio de Mataderos.En R.Sánchez Salineas(Comp.) El movimiento teatral comunitario argentino (pp. 167-218). CABA: Ediciones del CCC.

Sánchez Salinas, R. (2018) Las organizaciones culturales y su vínculo con el Estado: el caso del teatro comunitario mendocino. En M. S. Seguray A. V. Prato (Eds.),Estado, sociedad civil y politicas culturales. Rupturas y continuidades entre 2003 y 2017 (pp. 159-179). Buenos Aires: RCG Libros.

Sánchez Salinas, R. (2018). Resonancias actuales de la comunidad: el teatro comunitario argentino como espacio de recreación de lazos de pertenencia. Revista Question, 59, 1-18.

Segura, M. S., y Prato, V. (2018). Presentación. En Estado, sociedad civil y politicas culturales. Rupturas y continuidades en Argentina entre 2003 y 2017(pp. 13-20). Buenos Aires: RGC Ediciones.

Spilbarg, D.,y Saferstein, E. (2014). De la industria cultural a las industrias creativas: un análisis de la transformación del término y sus usos contemporáneos. Estudios de Filosofia Práctica e Historia de las Ideas, 2(16), 99-112.

Thwaites Rey, M., y Ouvina, H. (2012). La estatalidad Latinoamericana revisitada. Reflexiones e hipótesis alrededor del problema del poder político y las transiciones. En M. Thwaites Rey (Ed),El Estado en América Latina, continuidades y rupturas(pp. 51-92). Santiago de Chile: Ediciones Arcis.

Tovar y De Teresa, R. (1994). Modernización y politica cultural. México: FCE.

Wortman, A. (2002).Vaivenes del campo intelectual político cultural en la Argentina. En D.Mato, (coord.), Estudiosy otras prácticas intelectuales latinoamericanas en cultura y poder (pp. 327-338). Caracas: Consejo Latinoamericano de Ciencias Sociales y CEAP.

Wortman, A. (2005). El desafí de las politicas culturales en Argentina. Buenos Aires: CLACSO.

Wortman, A. (2017). Políticas culturales y legitimidad política en tiempos de crisis. El caso del Programa Puntos de Cultura de Argentina. Revista Cult. Rev,1(10), 138-160.

Yúdice, G. (2002). Contrapunteo estadounidense/latinoamericano de los estudios culturales. En D. Mato(coord.), Estudios y otras prácticas intelectuales latinoamericanas en cultura y poder (pp. 339-362). Caracas: CLACSO.

Yúdice, G. (2018). Prólogo. En V.Prato y S. Segura(Ed) Estado, sociedad civil y politicas culturales. Rupturas y continuidades en Argentina entre 2003 y 2017 (pp. 9-12). Buenos Aires: RGC Ediciones.

Urfalino, P. (1996). L'invention de la politiqueculturelle. París: La DocumentationFrançaise.

Zimmer, A. \& Toeppler, S. (1996). Cultural policies and the Welfare State: the cases of Sweden, Germany, and the United States. The Journal of Arts Management, Law and Society, (26)3, 167-193.

Zamorano, M. (2016).La transformación de las políticas culturales durante la primera década kirchnerista. Entre la hegemonía y la diversidad. Aposta, Revista de Ciencias Sociales, 70, 53-83.

\section{OTRAS FUENTES CONSULTADAS}

Página Web del Ministerio de Cultura de la Nación: https://www.cultura.gob.ar/

Página Web de Cultura en Números: https://culturaynumeros.wixsite.com/inicio

\section{ENTREVISTAS REALIZADAS}

Entrevista a Natalia Calcagno (Febrero 2018)

Entrevista a Francisco Romero (Junio 2017)

Entrevista a Jorge Pagés (Junio 2017)

Entrevista a Diego Benhabib (Febrero 2018) 


\section{Notas}

1 El presente artículo fue elaborado entre los meses de mayo de 2016 y diciembre del año 2017, por lo tanto sus referencias y reflexiones deben ser interpretadas a la luz de dicho marco temporal.

2 Estas últimas se utilizarán particularmente para la reconstrucción de los conflictos que se desarrollaron en el ambiente cultural a partir de la implementación de medidas específicas

3 En un ya clásico trabajo sobre políticas culturales, García Canclini (1987) identifica seis paradigmas culturales: mecenazgo liberal, tradicionalismo patrimonialista, estatismo populista, privatización neoconservadora, democratización cultural y democracia participativa

4 Volveremos luego sobre este punto

5 Se trata de los editores de revistas de Editoriales Independientes, Músicos Independientes, Trabajadores de la Danza y Productores Culturales Comunitarios

6 Es un colectivo de organizaciones sociales, culturales, medios de comunicación populares y fuerzas políticas que se unieron en apoyo a la cultura comunitaria. Ver: http://www.pueblohacecultura.org.ar/

7 Programa para soporte de artistas noveles de todo el país

8 El plan se propone establecer el acceso a la información y a la cultura como derechos fundamentales para el desarrollo pleno de los ciudadanos

9 Datos: tiene una capacidad para 1950 personas, 16 salas de ensayo, 40 salas de exposición, 2 terrazas, 18 vestíbulos, 6 auditorios y más de 1000 trabajadores

10 Es una agrupación cultural que surge en el año 2012, en Hurlingham, provincia de Buenos Aires, a partir de la iniciativa de Jorge Pagés y un grupo de jóvenes vinculados a distintas expresiones culturales

11 Para conocer detalles respecto del proceso de surgimiento del Consejo leer Pagés, J. (2018). Si te hace falta un consejo. El proyecto de Consejo Local de Cultura en Hurlingham. En A. Prato y M.S. Segura,Estado, sociedad civil y politicas culturales: rupturas y continuidades en Argentina entre 2003 y 2017. Buenos Aires: RGC Libros

12 Para más información sobre este tema se sugiere leer el artículo de Daniel Badenes disponible en: http://www.nuestras voces.com.ar/entendiendo-las-noticias/quien-escribe-la-nueva-ley-de-medios/

13 Recordamos el comunicado titulado "A la industria nacional no le da lo mismo", elaborado por diversas editoriales argentinas frente al balotaje de noviembre del 2015, donde se enfrentaban Mauricio Macri (Alianza Cambiemos) y Daniel Scioli (Frente para la Victoria)

14 El Movimiento de Espacios Culturales y Artísticos (MECA) ha realizado numerosas denuncias respecto del cierre de espacios culturales en la Ciudad de Buenos Aires bajo la gestión del PRO. A partir de la asunción de Cambiemos en el gobierno nacional se multiplicó esa dinámica de cierres, que ha sido denunciada incluso desde adentro de la gestión gubernamental, ya que existe la Ley 5240, sancionada el 18 de diciembre de 2014, que regula el funcionamiento de los centros culturales independientes

15 Esta proponeque los proyectos culturales puedan conseguir apoyo económico de las empresas, a cambio de que estas reduzcan sus contribuciones del impuesto de Ingresos Brutos

16 Datos obtenidos del Ministerio de Hacienda en su página Presupuesto Abierto (https://www.presupuestoabierto.gob .ar/sici/visualizaciones-gastos-y-recursos)

17 Se puede acceder por medio de este link: https://culturaynumeros.wixsite.com/inicio/que-es-cen

18 Se trata de los siguientes diez programas: Estudios e investigaciones históricas sobre Juan Domingo Perón, Fomento y ampliación de Derechos de la Cultura Popular, Fomento de las Industrias Culturales, Difusión de las manifestaciones político-culturales, Fomento y desarrollo para la identificación del Patrimonio Vivo y la Cultura Pública, Difusión, concientización y protección del Pat. Cultural del Museo de Bellas Artes, Desarrollo y fortalecimiento del sector cultural, Promoción de Políticas Culturales y Coop. Internacional, Fomento y apoyo económico a Bibliotecas Populares, Acceso igualitario y Promoción a los Derechos Culturales

19 La Ley 13.674, aprobada en el año 2007 y prorrogada en el 2012, establecía la utilidad pública y sujeto a expropiación de del inmueble (Centro Cultural Olga Vázquez), que funciona hace 14 años. La gobernadora de la provincia de Buenos Aires María Eugenia Vidal vetó dicha ley por decreto

20 Javier Grosman es un reconocido productor argentino que estuvo a cargo de la estética y la curaduría de los eventos más importantes durante la gestión kirchnerista

21 En una entrevista a la socióloga Natalia Calcagno (2018), quien trabajó en el área de cultura de nación desde el 2005 hasta el 2015, la funcionaria afirmó que la propuesta de orientar la política cultural en una integración Latinoamericana que no mire a Europa sino al Mercosur fue una directiva clara del poder ejecutivo, que impregnó fuertemente al gobierno de Néstor Kirchner. 
$22 \mathrm{Si}$ bien excede el recorte temporal de este trabajo, es importante destacar que a partir de septiembre de 2018 el Ministerio de Cultura de la Nación pasó a ser Secretaría de Cultura, dependiente del Ministerio de Educación, y redujo drásticamente su personal. 